\title{
Excessive Sweating is a Predictive Factor for Serious Consequences of Rhabdomyolysis Not Requiring Renal Replacement Therapies on Admission
}

\author{
Masaki Tago ${ }^{1 *}$, Naoko E Furukawa ${ }^{1}$, Yuka Naito ${ }^{1}$, Norio Fukumori ${ }^{2}$, Takashi Sugioka ${ }^{2}$ and Shu-ichi Yamashita ${ }^{1}$ \\ ${ }^{1}$ Department of General Medicine, Saga University Hospital, Saga, Japan \\ ${ }^{2}$ Community Medical Support Institute, Faculty of Medicine, Saga University, Saga, Japan
}

"Corresponding author: Masaki Tago, Department of General Medicine, Saga University Hospital, Saga University, 5-1-1 Nabeshima, Saga 849-8501, Japan, Tel: +81 95234 3238; Fax: +81 95234 2029; E-mail: tagomas@edu.cc.saga-u.ac.jp

Rec date: September 25, 2015 Acc date: October 19, 2015 Pub date: October 23, 2015

Copyright: (c) 2015 Tago M, et al. This is an open-access article distributed under the terms of the Creative Commons Attribution License, which permits unrestricted use, distribution, and reproduction in any medium, provided the original author and source are credited.

\begin{abstract}
Objective: Rhabdomyolysis is a serious syndrome which may lead to acute kidney injury and even death. However, reliable factors for predicting the prognosis of this condition, especially regarding patient's physical findings, remain to be clarified.

Methods: We retrospectively analyzed charts of patients who were clinically suspected of rhabdomyolysis with high serum creatine kinase on admission to the Department of General Medicine, Saga University Hospital, Japan from 2006-2013. Patients were divided into two groups, those progressing to serious consequences of death or acute kidney injury requiring renal replacement therapies during hospitalization (Group A) and those not progressing to such conditions (Group B). We compared background characteristics, symptoms, signs, and blood chemistry between both groups.
\end{abstract}

Results: A total of 53 Japanese patients were enrolled (men, 60.4\%; mean age 58.6 years). Group A consisted of five cases. According to univariate analysis, only the finding of excessive sweating on admission was related to the serious consequences (odds ratio 31.33, 95\% confidence interval 2.17-450.07, $\mathrm{p}<0.05$ ).

Conclusions: Excessive sweating on admission is a useful indicator to predict the prognosis of serious consequences of rhabdomyolysis.

Keywords: Rhabdomyolysis; Sweating; Neuroleptic malignant syndrome; Renal replacement therapies; Mortality

\section{Introduction}

Rhabdomyolysis is a rather common and potentially fatal condition characterized by muscle pain, myoglobinuria, and elevated serum creatine kinase (CK) levels resulting from the breakdown of skeletal muscle tissue $[1,2]$. A variety of diseases and other factors can cause rhabdomyolysis, including drugs, dehydration, trauma, infection, and collagen diseases [1-3]. Serum myoglobin plays an important role in establishing the pathologic conditions, particularly by causing acute renal tubular damage through deposition in the glomeruli after leakage into the systemic circulation. This can lead to acute renal failure, disseminated intravascular coagulation, and multiple organ failure [1,4-6].

In Japan, outpatients are entitled to free access to any medical institution, including university hospitals, without referral by their family doctor or general practitioner. Despite this situation, psychiatrists in Japan tend to have difficulty in referring their patients when they experience acute physical illnesses. This is because in Japan, especially in suburban cities, there is a shortage of hospitals that are available to treat serious physical illnesses in patients with severe psychiatric diseases. However, the presence of general medicine departments is invaluable in Japanese university hospitals, working as gatekeepers. A myriad of patients, including those with common and minor illnesses, mild trauma, and diverse physical complications of psychiatric illnesses, are treated at the outpatient clinic of the Department of General Medicine, Saga University Hospital, Japan, which increases the accessibility of this university hospital. In addition, large numbers of inpatients in our department experiencing severe and acute conditions are referred by doctors including psychiatrists at hospitals without adequate resources. Among such diseases, acute rhabdomyolysis is one of the most significant pathological conditions, many cases of which are introduced by psychiatrists having difficulty in finding appropriate institutions to which to refer their patients.

Predicting the prognosis of patients with acute rhabdomyolysis on admission, who do not currently require renal replacement therapies is difficult, and only aided by laboratory data such as serum CK (available on site) or serum myoglobin (usually only available after several days in Japan). However, predictive prognosis is imperative to prepare for the possibility of extremely severe consequences such as death, or acute kidney injury requiring renal replacement therapies. Patient background and etiology of acute rhabdomyolysis are considered to be useful predictive factors $[1,4]$. Considering our situation, in which we often receive patients from psychiatric hospitals, 
Citation: Tago M, Furukawa EN, Naito Y, Fukumori N, Sugioka T, et al., (2015) Excessive Sweating is a Predictive Factor for Serious Consequences of Rhabdomyolysis Not Requiring Renal Replacement Therapies on Admission. Gen Med (Los Angel) 3: 1000210. doi: $10.4172 / 2327-5146.1000210$

Page 2 of 6

neuroleptic malignant syndrome (NMS) is one of the major etiologies that has a high mortality rate (8.4\% within the first 30 days) [7]. Nevertheless, the reason patients with rhabdomyolysis are referred by psychiatric hospitals is not always NMS.

In this study, we investigated patients suspected of acute rhabdomyolysis with high serum CK in the Department of General Medicine, Saga University Hospital, Japan. Laboratory data and other clinical information, including physical findings, were assessed to elucidate predictive factors for the prognosis of patients with acute rhabdomyolysis not requiring renal replacement therapies on hospital admission.

\section{Materials and Methods}

\section{Patients}

All consecutive patients clinically suspected of acute rhabdomyolysis with high serum CK on admission to the Department of General Medicine, Saga University Hospital, Japan, from January 2006 to October 2013 were analyzed retrospectively. Patients whose high CK were caused by acute coronary syndrome, myocarditis or other diseases than rhabdomyolysis were excluded in this study. In addition, patients with rhabdomyolysis who required renal replacement therapies such as hemodialysis or continuous hemodiafiltration on admission were excluded, as well. Patients were divided into two groups, those developing serious consequences (death or acute kidney injury requiring renal replacement therapies) after their admission (Group A) and those not developing such conditions (Group B).

\section{Methods}

This was a retrospective chart-based study. Age, sex, duration from the appearance of symptoms to admission (days), length of hospital stay (days), clinical diagnosis, comorbid psychiatric diseases, kidney injury with or without the emergence of the need for renal replacement therapies during their post-admission hospital stay, symptoms, physical findings, and laboratory data on admission, worst laboratory results during hospitalization, and final post-admission outcome (discharged or deceased).

Symptoms and signs on admission were adopted from Levenson's criteria [8]. Data derived from patient charts included fever (body temperature $>38^{\circ} \mathrm{C}$ ), rigidity, tachycardia (pulse rate $>100 /$ minute), abnormalities in blood pressure (systolic blood pressure $<80 \mathrm{mmHg}$, $>140 \mathrm{mmHg}$, or diastolic blood pressure $>90 \mathrm{mmHg}$ ), tachypnea (respiratory rate $>20 /$ minute), disturbance of consciousness (Japan Coma Scale [JCS] $\geq 1$ ), [9] excessive sweating, and elevated white blood cell count $(>10,000 / \mu \mathrm{L})$. The presence of dehydration was determined by definite history of poor fluid intake, physical findings, and increased blood urea nitrogen (BUN) and creatinine serum levels. Laboratory data on admission included white blood cell $(/ \mu \mathrm{L}), \mathrm{CK}(\mathrm{IU} / \mathrm{L}), \mathrm{BUN}$ $(\mathrm{mg} / \mathrm{dL})$, creatinine $(\mathrm{mg} / \mathrm{dL})$, albumin $(\mathrm{g} / \mathrm{dL})$, sodium $(\mathrm{mEq} / \mathrm{L})$, and potassium $(\mathrm{mEq} / \mathrm{L})$ measurements. Highest levels of CK (IU/L), BUN $(\mathrm{mg} / \mathrm{dL})$, and creatinine $(\mathrm{mg} / \mathrm{dL})$ during hospitalization were also checked.

The primary etiological factor for rhabdomyolysis of each patient was determined according to Melli's classification [3]. NMS was not included as primary etiological factors because they usually exist concurrently with other major factors. The presence or absence of prescription antipsychotic or anti-parkinsonian drugs and statins were investigated. We reviewed all the patients to determine whether they fulfilled Levenson's criteria.

Descriptive statistics were used to present patient characteristics at baseline. Data are presented as mean ( \pm standard deviation), absolute numbers, or percentages. Differences in descriptive variables between patients with or without serious consequences were assessed using the chi-square test. The independent sample t-test was used to compare mean values between the two groups. Logistic regression analysis was performed to examine risk factors for serious consequences. In this model, sex, fever, rigidity, disturbance of consciousness, excessive sweating, and dehydration were used as explanatory variables. All calculations were performed using SPSS software (21.0.0, IBM). Statistical significance was set at $\mathrm{p}<0.05$.

This study was approved by the research ethical committee of our institute and adhered to the Principles of the Helsinki Declaration. The patients gave informed consent, and their anonymity was preserved.

\section{Results}

During the 8-year period from 2006-2013, 53 Japanese patients fulfilled the study inclusion criteria. Mean patient age was $58.6 \pm 17.2$ years and $60.4 \%$ were men. Patient characteristics, physical findings, and laboratory data are shown in Table 1 . The mean temperature, white blood cell count, CK, BUN, and creatinine levels on admission were higher than normal. The mean of the highest CK levels of all patients during hospitalization was higher than that on admission. The mean of serum electrolytes on admission showed no abnormalities.

\begin{tabular}{|l|c|c|}
\hline Variable & \multicolumn{2}{|c|}{ Mean \pm SD } \\
\hline Age on admission $(y)$ & $58.6 \pm 17.2$ & \\
\hline Sex & $32(60.4)$ \\
\hline Man, $n(\%)$ & $21(39.6)$ \\
\hline Woman, $n(\%)$ & $6.0 \pm 7.7$ \\
\hline Length before admission after the appearance of Symptoms (days) & $20.3 \pm 21.0$ \\
\hline Length of hospital stay (days) & \\
\hline Data on admission & \\
\hline
\end{tabular}


Citation: Tago M, Furukawa EN, Naito Y, Fukumori N, Sugioka T, et al., (2015) Excessive Sweating is a Predictive Factor for Serious Consequences of Rhabdomyolysis Not Requiring Renal Replacement Therapies on Admission. Gen Med (Los Angel) 3: 1000210. doi: $10.4172 / 2327-5146.1000210$

Page 3 of 6

\begin{tabular}{|c|c|}
\hline Body temperature $\left({ }^{\circ} \mathrm{C}\right)$ & $37.5 \pm 1.0$ \\
\hline Systolic blood pressure (mmHg) & $124 \pm 28$ \\
\hline Diastolic blood pressure $(\mathrm{mmHg})$ & $72 \pm 16$ \\
\hline Pulse rate (/minute) & $92 \pm 19$ \\
\hline Respiratory rate (/minute) & $22 \pm 6$ \\
\hline White blood cell $(/ \mu \mathrm{L})$ & $11130 \pm 4980$ \\
\hline Creatine kinase (IU/L) & $10150 \pm 18957$ \\
\hline Blood urea nitrogen (mg/dL) & $23.0 \pm 17.5$ \\
\hline Creatinine (mg/dL) & $1.13 \pm 0.92$ \\
\hline Albumin (g/dL) & $3.6 \pm 0.8$ \\
\hline Sodium (mEq/L) & $137 \pm 9$ \\
\hline Potassium (mEq/L) & $3.8 \pm 0.9$ \\
\hline \multicolumn{2}{|l|}{ Max data during hospitalization } \\
\hline Creatine kinase (IU/L) & $17273 \pm 31652$ \\
\hline Blood urea nitrogen (mg/dL) & $31.4 \pm 30.6$ \\
\hline Creatinine (mg/dL) & $1.46 \pm 1.58$ \\
\hline
\end{tabular}

Table 1: Characteristics of study population $(n=53)$.

Prescribed medications were the most common cause of rhabdomyolysis (29 patients, 54.7\%) followed by muscle disease, metabolic causes, and heat stroke/dehydration. Eleven patients fulfilled Levenson's diagnostic criteria for NMS, which included two of five patients in Group A. The other three patients in Group A resulted from muscle diseases, heat stroke/dehydration, or other disorders (Table 2).

\begin{tabular}{|l|c|c|c|}
\hline Category & No. of Patients $\mathbf{n = 5 3}(\%)$ & Fulfilled Levenson criteria $\mathbf{n = 1 1}$ & Group A $\mathbf{n}=\mathbf{5}$ \\
\hline Prescribed medicines & $29(54.7)$ & 10 & 2 \\
\hline Muscle diseases & $5(9.4)$ & 0 & 0 \\
\hline Metabolic causes & $5(9.4)$ & 0 & 1 \\
\hline Heat stroke/ Dehydration & $5(9.4)$ & 1 & 1 \\
\hline Others & & 0 & 0 \\
\hline Trauma & $3(5.7)$ & 0 & 0 \\
\hline Seizures & $2(3.8)$ & 0 & 0 \\
\hline Illicit drugs & $2(3.8)$ & 0 & 0 \\
\hline Exercise & $1(1.9)$ & 0 & \\
\hline
\end{tabular}

Table 2: Etiologic factors of rhabdomyolysis. ${ }^{\mathrm{a} G r o u p ~ A: ~ P a t i e n t s ~ w i t h ~ s e r i o u s ~ c o n s e q u e n c e s ~ o f ~ d e a t h ~ o r ~ a c u t e ~ k i d n e y ~ i n j u r y ~ r e q u i r i n g ~ r e n a l ~}$ replacement therapies such as hemodialysis or continuous hemodialysis and filtration. ${ }^{\mathrm{b}}$ Others: Sepsis, colitis, unknown.

On admission, six patients (11.3\% of all cases) had creatinine levels $>2 \mathrm{mg} / \mathrm{dL}$. During hospital stay, a further two cases showed creatinine levels $>2 \mathrm{mg} / \mathrm{dL}$. In addition, $3.8 \%$ of all patients required renal replacement therapies after their admission. The in-hospital mortality rate was 7.5\%. Two patients in Group A required renal replacement therapies (hemodialysis or continuous hemodiafiltration), and four patients died. Two patients in Group A were diagnosed with NMS, and four had psychiatric diseases (Table 3 ). 
Citation: Tago M, Furukawa EN, Naito Y, Fukumori N, Sugioka T, et al., (2015) Excessive Sweating is a Predictive Factor for Serious Consequences of Rhabdomyolysis Not Requiring Renal Replacement Therapies on Admission. Gen Med (Los Angel) 3: 1000210. doi: $10.4172 / 2327-5146.1000210$

Page 4 of 6

\begin{tabular}{|c|c|c|c|c|c|c|}
\hline Age (y) & Sex & Main complaint & Clinical diagnosis & Psychiatric disease & $\mathrm{HD}^{\mathrm{a}} / \mathrm{CHDF}^{\mathrm{b}}$ & $\begin{array}{l}\text { Outcome at the time } \\
\text { of discharge }\end{array}$ \\
\hline 36 & $M^{c}$ & Fatigue, Dyspnea & $\begin{array}{l}\text { Rhabdomyolysis of unknown } \\
\text { etiology }\end{array}$ & Autism & Performed & Death \\
\hline 77 & $W^{d}$ & Fever & Malignant syndrome & Bipolar disorder & Not performed & Death \\
\hline 75 & $M^{c}$ & Fatigue & Myopathy of unknown etiology & None & Not performed & Death \\
\hline 53 & $W^{d}$ & $\begin{array}{l}\text { Consciousness } \\
\text { disturbance }\end{array}$ & Malignant syndrome & Bipolar disorder & Performed & Recovery \\
\hline 89 & $M^{c}$ & Unable to complain & Acute choledochitis & Dementia & Not performed & Death \\
\hline
\end{tabular}

Table 3: Profile of five patients with serious consequences who underwent hemodialysis or continuous hemodialysis and filtration or died. ${ }^{\text {a HD: }}$ Hemodialysis. ${ }^{\mathrm{b}} \mathrm{CHDF}$ : Continuous Hemodialysis and Filtration. ${ }^{\mathrm{c}} \mathrm{M}$ : Man. ${ }^{\mathrm{d} W}$ : Woman.

According to univariate analysis, there were no significant betweengroup differences in sex, fever, muscle rigidity, and consciousness disturbance on admission. Only excessive sweating on admission was significantly associated with serious consequences during hospitalization (odds ratio 31.33 , 95\% confidence interval $2.17-$ 450.07, $\mathrm{p}=0.021)$ (Table 4).

\begin{tabular}{|c|c|c|c|c|c|}
\hline Symptoms on admission & Group $A^{a} n=5(\%)$ & Group $B^{b} n=48(\%)$ & OR $^{\mathrm{c}}$ & $95 \% \mathrm{Cl}^{\mathrm{d}}$ & $p$ value \\
\hline Man & $3(60.0)$ & $29(60.4)$ & 1.02 & $0.16-6.67$ & 1.000 \\
\hline Fever $\geq 38^{\circ} \mathrm{C}$ & $2(40.0)$ & $12(25.0)$ & 2.00 & $0.30-13.44$ & 0.599 \\
\hline Muscular rigidity and extrapyramidal symptoms & $1(20.0)$ & $14(29.1)$ & 0.61 & $0.06-5.92$ & 1.000 \\
\hline Consciousness disturbance & $1(20.0)$ & $21(43.8)$ & 0.32 & $0.03-3.09$ & 0.389 \\
\hline Excessive sweating & $2(40.0)$ & $1(2.1)$ & 31.33 & $2.17-450.07$ & 0.021 \\
\hline Dehydration & $4(80.0)$ & $19(39.6)$ & 6.11 & $0.63-58.88$ & 0.154 \\
\hline
\end{tabular}

Table 4: Univariate analysis between symptoms on admission and serious consequences (analyzed by chi-square test). ${ }^{\mathrm{a}} \mathrm{Group}$ A: Patient with serious consequences of death or acute kidney injury requiring renal replacement therapies such as hemodialysis or continuos hemodialysis and filtration. ${ }^{b}$ Group B: Patients without serious consequences of death or acute kidney injury requiring renal replacement therapies. ${ }^{c}$ OR: Odds ratio. ${ }^{\mathrm{d}} \mathrm{CI}$ : Confidence interval.

White blood cells and other blood chemistry (CK, BUN, creatinine, albumin, sodium, and potassium on admission. and peak CK, BUN, and creatinine), physical findings, duration of symptoms prior to admission, and length of hospital stay, had no significant association with serious consequences.
As expected, maximum serum BUN and creatinine were significantly associated with serious consequences (Table 5). According to the chi-square test, fever (body temperature $>38^{\circ} \mathrm{C}$ ) and excessive sweating had no significant association with other symptoms.

\begin{tabular}{|c|c|c|c|c|}
\hline Variable & Group $A^{a} n=5$ & Group $B^{b} n=48$ & $95 \% \mathrm{Cl}^{\mathrm{C}}$ & $p$ value \\
\hline Age on admission (y) & $66.0 \pm 21.2$ & $57.8 \pm 16.9$ & $-24.4-8.1$ & 0.318 \\
\hline $\begin{array}{l}\text { Length after symptom appeared before } \\
\text { admission (days) }\end{array}$ & $8.0 \pm 7.3$ & $5.8 \pm 7.8$ & $-9.6-5.1$ & 0.545 \\
\hline Length of stay (days) & $32.2 \pm 26.7$ & $19.1 \pm 20.2$ & $-32.8-6.5$ & 0.185 \\
\hline \multicolumn{5}{|l|}{ Examination on admission } \\
\hline Body temperature $\left({ }^{\circ} \mathrm{C}\right)$ & $38.0 \pm 2.3$ & $37.5 \pm 0.8$ & $-3.4-2.3$ & 0.635 \\
\hline Systolic blood pressure $(\mathrm{mmHg})$ & $111 \pm 12$ & $126 \pm 28$ & $-40-12$ & 0.271 \\
\hline Diastolic blood pressure $(\mathrm{mmHg})$ & $65 \pm 12$ & $72 \pm 16$ & $-23-8$ & 0.331 \\
\hline Pulse rate (/minute) & $104 \pm 23$ & $91 \pm 19$ & $-5-31$ & 0.148 \\
\hline
\end{tabular}


Citation: Tago M, Furukawa EN, Naito Y, Fukumori N, Sugioka T, et al., (2015) Excessive Sweating is a Predictive Factor for Serious Consequences of Rhabdomyolysis Not Requiring Renal Replacement Therapies on Admission. Gen Med (Los Angel) 3: 1000210. doi: $10.4172 / 2327-5146.1000210$

Page 5 of 6

\begin{tabular}{|c|c|c|c|c|}
\hline Respiratory rate (/minute) & $25 \pm 9$ & $21 \pm 6$ & $-3-10$ & 0.284 \\
\hline \multicolumn{5}{|l|}{ Data on admission } \\
\hline White blood cell $(/ \mu \mathrm{L})$ & $9700 \pm 3173$ & $11279 \pm 5132$ & $-6302-3144$ & 0.505 \\
\hline Creatine kinase (IU/L) & $10543 \pm 12186$ & $10109 \pm 19619$ & $-18492-17624$ & 0.962 \\
\hline Blood urea nitrogen $(\mathrm{mg} / \mathrm{dL})$ & $26.5 \pm 10.6$ & $22.6 \pm 18.1$ & $-20.5-12.7$ & 0.639 \\
\hline Creatinine (mg/dL) & $1.29 \pm 0.66$ & $1.12 \pm 0.95$ & $-1.05-0.70$ & 0.692 \\
\hline Albumin (g/dL) & $3.2 \pm 0.8$ & $3.7 \pm 0.8$ & $-0.3-1.2$ & 0.213 \\
\hline Sodium (mEq/L) & $135 \pm 11$ & $137 \pm 9$ & $-6-10$ & 0.627 \\
\hline Potassium (mEq/L) & $4.4 \pm 1.0$ & $3.8 \pm 0.9$ & $-1.5-0.2$ & 0.132 \\
\hline \multicolumn{5}{|c|}{ Max data during hospitalization } \\
\hline Creatine kinase (IU/L) & $32835 \pm 58331$ & $15652 \pm 28114$ & $-89154-54786$ & 0.549 \\
\hline Blood urea nitrogen $(\mathrm{mg} / \mathrm{dL})$ & $70.6 \pm 30.9$ & $27.4 \pm 27.8$ & $-69.7--16.8$ & 0.002 \\
\hline Creatinine (mg/dL) & $2.89 \pm 2.05$ & $1.31 \pm 1.47$ & $-3.01--0.14$ & 0.032 \\
\hline
\end{tabular}

Table 5: Univariate analysis between data or characteristics on admission and serious consequences (analyzed by independent sample t-test). ${ }^{a}$ Group A: Patients with serious consequences of death or acute kidney injury requiring renal replacement therapies such as hemodialysis or continuous hemodialysis and filtration. ${ }^{\mathrm{b}}$ Group B: Patients without serious consequences of death or acute kidney injury requiring renal replacement therapies. ${ }^{c} \mathrm{CI}$ : Confidence interval

\section{Discussion}

The present study results suggest that sweating could predict poor prognosis of rhabdomyolysis that did not require renal replacement therapies on admission. No previous reports have focused on signs and symptoms as predictive factors of serious consequences in such patients. As sweating is a physical finding that can be identified easily by a physician without any clinical instruments or laboratory tests, it is extremely valuable in the daily clinical setting. Dehydration has been reported to be one of the aggravating factors for renal failure in patients with rhabdomyolysis [10,11]. Theoretically, sweating exacerbates dehydration, which can subsequently make the prognosis of rhabdomyolysis poorer. However, in this study, the relationship between dehydration and aggravation of rhabdomyolysis, and between sweating and dehydration, were not statistically significant by univariate analysis. Autonomic dysfunction is considered to be one of the causes of sweating in rhabdomyolysis, especially in NMS, [12] which indicates the possibility that autonomic dysfunction is a more plausible cause of the association between sweating and aggravation of rhabdomyolysis than dehydration.

In this study, there was no significant difference between Groups A and $\mathrm{B}$ for age, sex, and serum creatinine and CK on admission and peak CK during hospitalization. Serum albumin $<3.3 \mathrm{mg} / \mathrm{dL}$, acidosis, prothrombin time $<82 \%$, and maximum CK level $>12,750$ IU/L were reported to be predictors of acute kidney injury in a study in patients with CK levels $>5000$ IU/L [6]. Another study in patients with rhabdomyolysis caused by illicit drug and alcohol use showed a correlation between maximum CK and serum creatinine level during hospitalization without showing a correlation between the introduction of hemodialysis and death [3]. In addition, in a study on the predictive factors of the prognosis of acute kidney injury in rhabdomyolysis, Gearoid et al. reported that age, sex, causes of rhabdomyolysis, serum creatinine, $\mathrm{CK}$, phosphate, calcium and
$\mathrm{HCO}^{3-}$ levels on admission were independent predictive factors for death or the need for renal replacement therapies [13]. However, in the current study, there were no statistical differences between age, sex, serum creatinine, CK, albumin on admission, and maximum CK during hospitalization, between Groups A and B. Although serum creatinine and CK levels on admission had been expected to be associated with the prognosis of rhabdomyolysis considering the pathophysiology, serious consequences might have been prevented in our study because of early and sufficient rehydration on admission, resulting in no significant differences for these parameters between Groups A and B.

The mortality rate of rhabdomyolysis was $7.5 \%$ in this study, but ranged from 1.7 to $59 \%$ in previous reports in patients with different backgrounds $[1,2,5,14]$. A study in patients with rhabdomyolysis caused by illicit drug and alcohol use showed mortality was 3.4\% [3] Other studies in patients with rhabdomyolysis treated in the intensive care unit showed the mortality rate was 59\% with renal failure and $22 \%$ without renal failure $[15,16]$. Because half the cases in our study were caused by prescribed drugs, the mortality rate was different from that for rhabdomyolysis caused by illicit drugs and alcohol. In addition, patients who needed renal replacement therapies on admission (and were thus expected to have a higher mortality rate), were not included in our study; this might explain the lower mortality rate in our study compared with that reported in the study in patients treated in intensive care units. In our study, $3.7 \%$ of patients required the introduction of renal replacement therapies, which was lower than that in previous studies $(8.0-9.5 \%)[6,13,17]$. The lower frequency in our study might be because we excluded patients who required renal replacement therapies on admission.

In previous studies, the predominant causes of rhabdomyolysis were illicit drugs, alcohol, prescribed drugs, myopathies, trauma, NMS and seizure disorders, and prolonged immobility $[3,6,13]$. The main causes 
Citation: Tago M, Furukawa EN, Naito Y, Fukumori N, Sugioka T, et al., (2015) Excessive Sweating is a Predictive Factor for Serious Consequences of Rhabdomyolysis Not Requiring Renal Replacement Therapies on Admission. Gen Med (Los Angel) 3: 1000210. doi: $10.4172 / 2327-5146.1000210$

Page 6 of 6

in our study were prescribed drugs, which accounted for $55 \%$ of cases, followed by myopathies, metabolic diseases, and heat stroke/ dehydration. Our department mainly treats patients with diseases of general internal medicine, with a low frequency of illicit drug use and trauma, which resulted in prescribed drugs being the main cause. Our department belongs to the university hospital, which facilitates our ability to treat patients referred to us by psychiatrists. This fact leads psychiatrists in other local hospitals to refer their patients when they experience serious diseases of internal medicine. As a result, we have many opportunities to treat patients with rhabdomyolysis caused by psychiatric medicines. The causes of rhabdomyolysis vary according to patient background and so does the probability of serious consequences. This is the reason it is imperative to take patient background into consideration when we predict the prognosis of rhabdomyolysis.

NMS can be a fatal disease which occurs in $0.07-3.23 \%$ of patients using antipsychotic drugs, with a considerably high mortality rate of about $10 \%[7,18,19]$. There were 11 cases $(20.8 \%)$ fulfilling Levenson's criteria in this study [8]. Of those patients, only three (27\%) had a serious prognosis. Because sweating is one of the important symptomatic factors of Levenson's criteria, [8] it may have some relationship with the aggravation of rhabdomyolysis with NMS. Our study, however, did not show a significant difference between the rate of patients fulfilling Levenson's criteria between Groups A and B.

The present study has several limitations. This study was a retrospective chart review. Although we presumed that physicians in our department were in agreement about treatments including hydration and the introduction of renal replacement, these treatments were provided in an arbitrary manner. In addition, the sample size was too small to conduct multivariate analysis, which made it impossible to adjust for various confounding factors such as age, fever, and dehydration. With regard to laboratory data, we didn't analyze $\mathrm{pH}$, prothrombin time, or calcium, because they had rarely been investigated on admission. Although urinary myoglobin was reported to be a useful marker of predicting poor prognosis, [1,3] we didn't analyze this because it usually takes several days to obtain the result in our institute, which negates its use in predicting prognosis on admission.

\section{Conclusion}

In this study, for the first time, we clarified the presence of excessive sweating on admission is a useful factor to predict the serious prognosis of death or acute kidney injury requiring renal replacement therapies in patients who are hospitalized because of rhabdomyolysis not requiring renal replacement therapy on admission.

\section{References}

1. Khan FY (2009) Rhabdomyolysis: a review of the literature. Neth J Med 67: 272-283.

2. Zimmerman JL, Shen MC (2013) Rhabdomyolysis. Chest 144: 1058-1065.

3. Melli G, Chaudhry V, Cornblath DR (2005) Rhabdomyolysis: an evaluation of 475 hospitalized patients. Medicine (Baltimore) 84: 377-385.

4. Chatzizisis YS, Misirli G, Hatzitolios AI, Giannoglou GD (2008) The syndrome of rhabdomyolysis: complications and treatment. Eur J Intern Med 19: 568-574.

5. Bosch X, Poch E, Grau JM (2009) Rhabdomyolysis and acute kidney injury. N Engl J Med 361: 62-72.

6. Rodríguez E, Soler MJ, Rap O, Barrios C, Orfila MA, et al. (2013) Risk factors for acute kidney injury in severe rhabdomyolysis. PLoS One 8: e82992.

7. Nielsen RE, Wallenstein Jensen SO, Nielsen J (2012) Neuroleptic malignant syndrome-an 11-year longitudinal case-control study. Can J Psychiatry 57: 512-518.

8. Levenson JL (1985) Neuroleptic malignant syndrome. Am J Psychiatry 142: 1137-1145.

9. Ohta T, Waga S, Handa H, Saito I, Basugi N, et al. (1975) New grading of level of consciousness in acute stage. Surg Cereb Stroke 3: 61-68.

10. Vanholder R, Sever MS, Erek E, Lameire N (2000) Rhabdomyolysis. J Am Soc Nephrol 11: 1553-1561.

11. Harriston S (2004) A review of rhabdomyolysis. Dimens Crit Care Nurs 23: $155-161$.

12. Pelonero AL, Levenson JL, Pandurangi AK (1998) Neuroleptic malignant syndrome: a review. Psychiatr Serv 49: 1163-1172.

13. McMahon GM, Zeng X, Waikar SS (2013) A risk prediction score for kidney failure or mortality in rhabdomyolysis. JAMA Intern Med 173: 1821-1828.

14. Keltz E, Khan FY, Mann G (2014) Rhabdomyolysis. The role of diagnostic and prognostic factors. Muscles Ligaments Tendons J 3: 303-312.

15. de Meijer AR, Fikkers BG, de Keijzer MH, van Engelen BG, Drenth JP (2003) Serum creatine kinase as predictor of clinical course in rhabdomyolysis: a 5-year intensive care survey. Intensive Care Med 29: 1121-1125.

16. Woodrow G, Brownjohn AM, Turney JH (1995) The clinical and biochemical features of acute renal failure due to rhabdomyolysis. Ren Fail 17: 467-474.

17. Brown CV, Rhee P, Chan L, Evans K, Demetriades D, et al. (2004) Preventing renal failure in patients with rhabdomyolysis: do bicarbonate and mannitol make a difference? J Trauma 56: 1191-1196.

18. Anglin RE, Rosebush PI, Mazurek MF (2010) Neuroleptic malignant syndrome: a neuroimmunologic hypothesis. CMAJ 182: E834-838.

19. Gillman PK (2010) Neuroleptic malignant syndrome: mechanisms, interactions, and causality. Mov Disord 25: 1780-1790. 